\title{
Pure hydroxyapatite as a substitute for enamel in erosion experiments
}

Ronay, Franz C ; Wegehaupt, Florian J ; Becker, Klaus ; Wiedemeier, Daniel B ; Attin, Thomas ; Lussi, Adrian ; Steiger-Ronay, Valerie

\begin{abstract}
OBJECTIVES The study evaluated the suitability of pure crystalline hydroxyapatite (HA) as a substitute for human $(\mathrm{H})$ and bovine $(\mathrm{B})$ enamel in erosion experiments. METHODS Human and bovine enamel and hydroxyapatite specimens $(\mathrm{n}=18 \mathrm{each}$ ) were submitted to demineralisation by $\mathrm{HCl}$ at $\mathrm{pH}$ values of $3.0,2.6$ and 2.0 (1.0, 2.5, and $10 \mathrm{mmol} / \mathrm{l}$ concentration). Specimens were eroded in a superfusion chamber for $7 \mathrm{~min}$ (flow rate: $1 \mu \mathrm{l} / \mathrm{s}$ ). Multiple fluid samples were taken per specimen throughout the exposure period. The dissolved calcium content was measured using a colorimetric assay with Arsenazo III reagent, to serve as surrogate for erosive substance loss. Additionally, erosive tissue loss was quantified by profilometry. Differences in erosive substance loss were assessed by the Kruskal-Wallis and Conover's posthoc test. RESULTS Good agreement in erosive substance loss between HA and human specimens was found at $\mathrm{pH}$ 2.6, as no statistically significant difference and considerable overlap of their respective interquartile ranges was observed. At pH 2.0 and 3.0, a statistically significant difference between HA and human and bovine enamel was determined. HA consistently tended to have lower mean dissolution rates, with a maximum deviation from human enamel of $-16 \%$ at $\mathrm{pH} 3$. It displayed lower specimen variability with an average coefficient of variation of $17 \%$, compared to $25 \%(\mathrm{H})$ and $25 \%$ (B), respectively. CONCLUSIONS Crystalline hydroxyapatite may not be suitable for full substitution of biologic enamel in erosion experiments focusing on absolute measurement values, but is useful for establishing consistent relative trends between erosive agents due to biosimilar erosion behavior and lower experimental variability. This is especially true for preliminary studies where approval for use of biological samples is restricted. CLINICAL SIGNIFICANCE Crystalline hydroxyapatite is a useful substitute of human or bovine enamel in experiments studying chemical aspects of dental erosion.
\end{abstract}

DOI: https://doi.org/10.1016/j.jdent.2019.04.002

Posted at the Zurich Open Repository and Archive, University of Zurich

ZORA URL: https://doi.org/10.5167/uzh-181511

Journal Article

Accepted Version

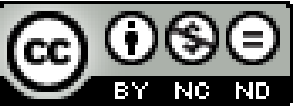

The following work is licensed under a Creative Commons: Attribution-NonCommercial-NoDerivatives 4.0 International (CC BY-NC-ND 4.0) License.

Originally published at:

Ronay, Franz C; Wegehaupt, Florian J; Becker, Klaus; Wiedemeier, Daniel B; Attin, Thomas; Lussi, Adrian; SteigerRonay, Valerie (2019). Pure hydroxyapatite as a substitute for enamel in erosion experiments. Journal of Dentistry, 84:89-94. 
DOI: https://doi.org/10.1016/j.jdent.2019.04.002 


\section{Pure hydroxyapatite as a substitute for enamel in erosion experiments}

Franz C. Ronay*, Florian J. Wegehaupt**, Klaus Becker**, Daniel B. Wiedemeier***, Thomas Attin**, Adrian Lussi****, Valerie Steiger-Ronay**

* Clinic of Preventive Dentistry, Periodontology and Cariology, Center of Dental Medicine, University of Zurich, Switzerland and Medical University of Vienna, Austria

** Clinic of Preventive Dentistry, Periodontology and Cariology, Center of Dental Medicine, University of Zurich, Switzerland

*** Statistical Services, Center of Dental Medicine, University of Zurich, Switzerland **** Department of Preventive, Restorative and Pediatric Dentistry, University of Bern, Switzerland

Short title: Hydroxyapatite as substitute for enamel

Keywords: demineralization, human, bovine, hydrochloric acid, profilometry, calcium

\section{Corresponding author:}

Dr. Valerie Steiger-Ronay

Clinic of Preventive Dentistry, Periodontology and Cariology

Plattenstrasse 11

8032 Zürich

Switzerland

Phone: $\quad+41446343284$

Fax: $\quad+41446344308$

Email: $\quad$ valerie.steiger@zzm.uzh.ch

Declarations of interest: none. 


\title{
Pure hydroxyapatite as a substitute for enamel in erosion experiments
}

\author{
Abstract \\ Objectives: \\ The study evaluated the suitability of pure crystalline hydroxyapatite (HA) as a substitute for \\ human $(\mathrm{H})$ and bovine $(\mathrm{B})$ enamel in erosion experiments.
}

\section{Methods:}

Human and bovine enamel and hydroxyapatite specimens $(n=18$ each) were submitted to demineralisation by $\mathrm{HCl}$ at $\mathrm{pH}$ values of 3.0, 2.6 and $2.0(1.0,2.5$, and $10 \mathrm{mmol} / \mathrm{l}$ concentration). Specimens were eroded in a superfusion chamber for 7 min (flow rate: 1 $\mu 1 / \mathrm{s})$. Multiple fluid samples were taken per specimen throughout the exposure period. The dissolved calcium content was measured using a colorimetric assay with Arsenazo III reagent, to serve as surrogate for erosive substance loss. Additionally, erosive tissue loss was quantified by profilometry. Differences in erosive substance loss were assessed by the Kruskal-Wallis and Conover's posthoc test.

\section{Results:}

Good agreement in erosive substance loss between HA and human specimens was found at $\mathrm{pH} 2.6$, as no statistically significant difference and considerable overlap of their respective interquartile ranges was observed. At $\mathrm{pH} 2.0$ and 3.0, a statistically significant difference between HA and human and bovine enamel was determined. HA consistently tended to have lower mean dissolution rates, with a maximum deviation from human enamel of $-16 \%$ at $\mathrm{pH}$ 3. It displayed lower specimen variability with an average coefficient of variation of $17 \%$, compared to $25 \%(\mathrm{H})$ and $25 \%(\mathrm{~B})$, respectively.

\section{Conclusions:}

Crystalline hydroxyapatite may not be suitable for full substitution of biologic enamel in erosion experiments focusing on absolute measurement values, but is useful for establishing consistent relative trends between erosive agents due to biosimilar erosion behavior and lower experimental variability. This is especially true for preliminary studies where approval for use of biological samples is restricted.

\section{Clinical significance:}

Crystalline hydroxyapatite is a useful substitute of human or bovine enamel in experiments studying chemical aspects of dental erosion. 


\section{Introduction}

Dental erosion is defined as the irreversible loss of dental hard tissue due to chemical dissolution by acids without bacterial involvement [1]. Dental erosion is used in cases of surface loss caused solely by extended exposure to acids (chemical process). Erosive tooth wear is the outcome of chemical-mechanical forces where the softened surface will be removed by abrasive forces, and presents a condition of growing concern with a high prevalence [2]. At least $10 \%$ of adolescents and adults in developed countries present with erosive tooth wear, with some sub-populations having prevalence rates of 80-100\% [3].

The occurrence and severity of erosive tooth wear is influenced by lifestyle factors, such as of consumption of acidic beverages like soft drinks [4], and diseases resulting in dental exposure to intrinsic acid (e.g. bulimia, gastroesophageal reflux) [5]. The prevalence of erosive tooth wear has increased over the last decades [6], while the penetration of intrinsic acid disorders is at a high level: in European populations, gastroesophageal reflux disease (GERD) prevalence stands at $>17 \%$ [7], and female bulimia prevalence has been stable at $1-4 \%[8,9]$. This highlights the importance of research into causation and prevention of this relevant health issue. On the causation side, factors influencing erosive tooth wear are being extensively studied both in vitro and in vivo [10]. In the context of prevention, approaches such as protective gels, mousses and dentifrices have been investigated [11-13].

The majority of studies apply in vivo research due to higher controllability of experimental conditions, as well as financial and ethical considerations incurred by in vivo studies. Furthermore, collecting and preparing human enamel specimens for in vitro studies can be challenging. Procurement of teeth in suitable numbers and quality, as well as preparing smaller specimens from them without damage takes up time and effort. Therefore, bovine enamel has been considered as a suitable substitute, as it is considered to have relatively similar characteristics in erosion studies [14]. However, not all research institutions have access to bovine material; the preparation, while slightly simpler than with human specimens, is still time-intensive. Finally, standardization of specimens of any biological origin is made difficult by natural variation. This natural variation can be caused by sampling from different tooth types or different regions of the same tooth, and of course individual variation between the original biological donors.

Given that enamel consists of over $90 \mathrm{wt} \%$ crystalline hydroxyapatite (HA) $[15,16]$, pure HA specimens of inorganic origin are worth considering as a potential substitute for enamel in erosion experiments. This would be attractive from two standpoints. Solid HA of $>99 \%$ 
purity in various forms is readily available from laboratory suppliers, which simplifies procurement. Secondly, HA disks or pills should in theory be easily standardisable, given that they are industrially manufactured to customisable specifications. Overall, this would simplify future basic research and make more reproducible benchmarking of erosion effects possible without requiring the procurement and preparation of a large number of biological specimens to overcome natural variability.

Therefore, this study aims to investigate whether pressed pure crystalline hydroxyapatite can act as a substitute for enamel in erosion studies when using $\mathrm{HCl}$ as the erosive agent in a superfusion model that is designed to mimic flow of an erosive agent in vivo under transient exposure conditions (such as initial contact with refluxate or drinking). The null-hypothesis was that HA would react similar to human and bovine enamel within measurement tolerance but with decreased variability.

\section{Materials and Methods}

\section{Specimen preparation}

Human and bovine enamel specimens $(n=18$ each) were prepared for acid exposure using the method described by Attin et al. [20], and following approved protocols. Disks of $3 \mathrm{~mm}$ diameter and $2 \mathrm{~mm}$ thickness were drilled from the crown of permanent incisors using a water-cooled hollow-core drill, and embedded in acrylic resin blocks of $6 \mathrm{~mm}$ diameter. The surface was smoothed in a rotating grinder at $100 \mathrm{rpm}$ for $40 \mathrm{~s}$ with a sequence of \#2000- and \#4000-grit waterproof silicon carbide paper under constant water cooling. Further, crystalline hydroxyapatite (HA) specimens of $99 \%$ purity and $3.1 \mathrm{~g} / \mathrm{cm}^{3}$ density (HAD130109 Solid Disk; HiMed, Old Bethpage, USA) were sourced and prepared in a similar way. HA specimens $(\mathrm{n}=18)$ with a diameter of $3 \mathrm{~mm}$ were drilled from $25 \mathrm{~mm}$ disks, were embedded in resin, and ground in the same manner as the human and bovine material.

\section{Controlled erosion using superfusion}

Susceptibility of the substrates to erosion under different acidity levels was measured by superfusion with a steady laminar flow of $\mathrm{HCl}$ at different $\mathrm{pH}$ values, succeeded by a colorimetric assay for calcium levels using the Arsenazo III reagent [17]. Given the typical range of $\mathrm{pH}$ levels of refluxate (which can range between $\mathrm{pH} 1-6$, with typical means around $\mathrm{pH}$ 2-3 for GERD sufferers $[18,19]$ and the most accurate measurement range of the assay, $\mathrm{pH} 3.0,2.6$ and 2.0 provided a comprehensive test range.) 
Specimens were placed in a superfusion apparatus for exposure to $1 \mu \mathrm{l} / \mathrm{s}$ of $\mathrm{HCl}$ over the surface for a total of 7 minutes. This apparatus was custom-built (PPK laboratory, University of Zurich, Switzerland) and consisted of a jig for fixing the specimens within small chambers of about $0.94 \mathrm{~mm}^{3}$ volume ( $2 \mathrm{~mm}$ diameter and $0.3 \mathrm{~mm}$ height), with two orifices of $0.2 \mathrm{~mm}$ radius through which acidic solution was pumped in and out to maintain laminar, bubble-free

flow. Each of the chambers was connected to a single tube of a multichannel pump (IPC; Ismatec, Glattbrugg, Switzerland), allowing for controlled testing of multiple specimens at once [21]. An initial conditioning period of 2 minutes of acid flow over the specimens was implemented to avoid nonlinearities at the beginning of the run. Transient sources of error such as initial surface contamination, remaining air bubbles in the test chamber etc. were mitigated in this way. After this conditioning period, the acidic solution and dissolved calcium were captured every $30 \mathrm{~s}$ in rows of a collection dish. This yielded a total of ten 30 $\mu 1$ sample volumes - and hence 10 data points - per specimen and $\mathrm{pH}$. The dissolution protocol was repeated for each specimen with three solutions of ascending magnitude of

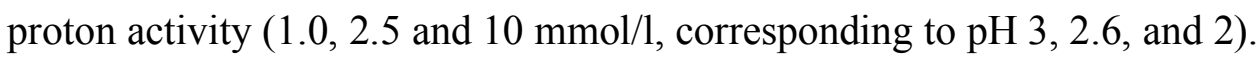

\section{Calcium dissolution measurement}

For the colorimetric measurement of calcium content, a protocol as described by Attin et al. [17] was followed. $10 \mu 1$ of each of the $30 \mu 1$ samples of acidic solution were pipetted from the superfusion collection dish into a clear 96-well microplate and mixed with $100 \mu$ of the indicator reagent solution consisting of $100 \mathrm{mmol} / \mathrm{l}$ Imidazol buffer $(\mathrm{pH} 6.5)$ and $0.12 \mathrm{mmol} / 1$ Arsenazo III (Fluitest Ca-A-II; Analyticon, Lichtenfels, Germany). The indicator changed colour in the presence of calcium, thereby shifting the absorption spectrum of the sample at the photometry wavelength of $650 \mathrm{~nm}$ in proportion to calcium mass. Standard solutions of known calcium concentration were prepared by geometrically decreasing dilution. Finally, absorption of the standard and experimental samples was measured in a microplate reader (Spectramax; Bucher Biotec, Basel, Switzerland). The standard measurements yielded a calibration curve for calcium concentration, from which the calcium content of the erosion solution samples was derived.

\section{Additional calibration measurements}

While calcium measurement was the main test metric, the phosphate concentrations were measured for a subset of samples $(n=7)$ so as to ascertain a consistent release ratio of calcium and phosphate. Phosphate concentration was measured using colorimetry with 
malachite green as the complexing agent. In order to create the reagent as described in Hannig et al. [22] $0.045 \mathrm{mg}$ Malachite green was dissolved in $100 \mathrm{ml} \mathrm{H}_{2} 0$, admixed to $12.69 \mathrm{~g}$ of ammonium molybdate dissolved in $300 \mathrm{ml} \mathrm{HCl}(4 \mathrm{~mol} / \mathrm{l})$, and then stirred for 30 minutes and passed through $0.22 \mu \mathrm{m}$ pore filtration. Colorimetry measurements then proceeded in similar fashion to the calcium assay via a microplate reader and a separately calculated standard absorption curve at $650 \mathrm{~nm}$ wavelength.

\section{Profilometry}

The profiles of sample HA disks after acid exposure were qualitatively compared to those of human enamel specimens to check for any gross difference in physical erosion behaviour. A stylus profilometer (Perthometer S2; Mahr, Göttingen, Germany) was used to determine the depth of the eroded region of individual specimens ( $n=2$ per type) to compare the surface characteristics of pure HA specimens with those of biological enamel. The automated stylus recorded the profile of the $3 \mathrm{~mm}$ specimens along 12 tracks over the surface at an upper-limit resolution of $10 \mathrm{~nm}$. An approximate estimate of volume lost could be made by taking average erosive lesion depth over the surfaces for separate validation of the calcium dissolution measurement.

\section{Statistics}

The statistical software $\mathrm{R}$ was used for all data analyses [23]. The calcium release measurements were plotted and descriptive statistics calculated. The mean coefficient of variation (\%) was computed to determine material variability. Due to violations of ANOVA assumptions (e.g. heteroscedasticity) a non-parametric approach was employed to compare the central tendency between the materials. At each $\mathrm{pH}$ level, the Kruskal-Wallis rank sum test, followed by pair-wise comparisons using Conover's test [24], were used to test for significant differences of calcium release between HA, bovine and human specimens. The significance level $\alpha$ was set to 0.05 .

\section{Results}

\section{Calcium dissolution measurements}

The calcium dissolution rate was constant over time, indicating a linear progression of erosion in all specimen groups. The average slope of the time-dissolution curve was close to zero, i.e. approximately the same amount of calcium was dissolved every $30 \mathrm{~s}$. A graphical 
comparison of calcium dissolution rates at all three $\mathrm{pH}$ levels is shown for overview in Figure 1 .

Table 1 summarizes the mean calcium release values, which were similar within the tested $\mathrm{pH}$ for all three specimen types. Pairwise statistical comparisons revealed no significant difference between HA or bovine specimens and human specimens, except for HA at $\mathrm{pH} 3$ and $\mathrm{pH} 2(\mathrm{p}<0.05)$. The mean coefficient of variation was lowest for the HA specimens with only $17 \%$, while human and bovine specimens presented with values of $25 \%$ and $25 \%$ (Table 1). Table 2 lists the distribution of calcium release values for a numerical comparison of the quartile ranges.

\section{Phosphate dissolution measurements}

The measurements of phosphate dissolution in the calibration subset yielded mean calcium to phosphate release ratios of $1.57(\mathrm{H}), 1.47(\mathrm{~B})$, and $1.60(\mathrm{HA})$, which were consistent across all three $\mathrm{pH}$ levels with a variance below $10 \%$ for each specimen type.

The level of proton consumption in the acidic superfusion solution - as reflected by the difference in $\mathrm{pH}$ of the solution exposed to the sample compared to the original source solution $\mathrm{pH}$ - was consistently under $20 \%$ even at the lowest $\mathrm{pH}$ level tested.

\section{Profilometry}

The profiles of the HA specimens after acid exposure were qualitatively compared to those of human enamel specimens in order to check for any gross difference in physical erosion behaviour. In both human enamel and HA inclines in the direction of the acid flow could be observed. HA specimens, however, displayed larger discontinuities on the order of 1-10 $\mu \mathrm{m}$ in the curved erosion profile. This is illustrated by isolated mini-peaks, as can be seen in the sample profilometric difference curve (Figure 2).

\section{Discussion}

Assessing the amount of calcium released from enamel as a surrogate measurement of dental enamel dissolution is an accepted research method [10]. Given that it has been demonstrated that the dissolution of calcium through a fully dissociating acid like $\mathrm{HCl}$ proceeds at an approximately linear rate over time [22], each measurement can be seen to reflect the overall dissolution rate and therefore the susceptibility to erosion of the materials. 
Factors for a functional comparison of $H A$ and human enamel

When evaluating HA as a potential substitute for natural enamel specimens in erosion studies, four factors need to be considered, which are listed roughly in descending order of importance:

- consistency

- similar chemical behaviour to enamel in the experimental context

- comparative ease of preparation and standardization

- optimally - but also optionally - similar physical characteristics and biological interactions

\section{Consistency}

Commercially available HA specimens should behave consistently between experiments, and ideally between manufacturers - otherwise erosion studies performed with HA specimens may not replicate. As shown in Table 1, consistency within a specimen range is indeed the case. An average variability of $17 \%$ was observed with the HA specimens at different $\mathrm{pH}$ levels - lower than for both biological specimen types. Furthermore, the HA erosion proceeded linearly over the experimental time range, just like for specimens of biologic origin.

\section{Chemical similarity}

Similarity of surface chemistry narrowly refers to the response of the smooth material surface to acidic $\mathrm{pH}$ in this study. Many studies on erosion restrict themselves to this domain of specimen response. Hence simple surface erosion response is a good proxy for a large range of studies that enamel specimens are used for.

One can see in Figure 1 and Table 2 that the interquartile range of measured calcium dissolution of the HA specimens overlaps well with the range of the human and bovine specimens at $\mathrm{pH} 2.0$ and $\mathrm{pH} 2.6$, whereas the $\mathrm{pH} 3.0$ measurement range undershoots that of human and bovine enamel specimens. The median dissolution rate of $\mathrm{HA}$ is about $16 \%$ less than compared to human material at this $\mathrm{pH}$. Table 1 shows that statistical significant differences between the HA and human series were detectable at $\mathrm{pH} 2.0$ and 3.0. This matches experiences from a past study by Shellis et al [25] which also found a lower dissolution tendency of HA in comparison to human dental tissues. While the cited study applied a different methodology using a pH-stat system to study the kinetics of dissolution of 
HA, human enamel and human dentin over a longer period of time (30 min), our study focused on the differences between human enamel in comparison to bovine enamel or synthetic HA discs applying a superfusion model, which should mimic the transient flow of an erosive agent over the specimen's surfaces in a shorter period of time $(7 \mathrm{~min})$.

Two compositional differences of the enamel can be identified which may play a role in deviation in surface dissolution rate of pure HA disks: protein content, and nonstoichiometric apatite components in the enamel. The protein content in enamel (human enamel being $<1 \%$ protein by weight [26]) of course is not reflected in the HA discs, which could play a role in the high variability of biological enamel. Non-stoichiometric substitution of other substances and groups such as carbonate and magnesium may be an even more important factor. The carbonated hydroxyapatite is known to be more soluble in acid than hydroxyapatite, which in turn is more soluble than fluorapatite $[27,28]$. In a past experiment with powdered enamel [29] the presence of carbonate groups in enamel was implicated in increased solubility rates over those expected from stoichiometric HA.

Phosphate dissolution measurements in the subset did not impact the calcium measurements: phosphate dissolution rate proceeded in lockstep with calcium dissolution rate and confirmed the trend seen in the overall test series. The calcium-to-phosphate ratios were constant across $\mathrm{pH}$ levels for each specimen type, and in line with theoretical expectations (assumed to be around 1.62 for enamel [29]).

Previously, a literature review by Yassen et al. [30] summarised research on the use of bovine specimens as substitutes for human enamel and dentin. 68 studies were included, covering a range of research categories where human teeth can be substituted with bovine material. The comparisons set a precedent of testing for clinically relevant differences, and allowed for substitution of in erosion experiments as long as no difference was detected [31] or if the difference was small and could be accounted for with a conversion constant [32]. Under this standard, preformed pure HA disks would not be suitable for direct substitution if maximum accuracy of absolute values is desired - one would have to take into account downward deviations of up to $16 \%$. However, due to higher self-consistency between experiments they may be useful if it is desired to quickly establish relative trends in dissolution that result from varying $\mathrm{pH}$ levels or experimental conditions. More expansive testing could establish correction factors with narrower confidence intervals at each $\mathrm{pH}$ level, which would allow 
direct substitution for absolute measurements.

\section{Ease of preparation and standardization}

Ease of preparation was one of the original motivations for using HA specimens, and therefore worth a brief evaluation. For HA specimens these properties depend on the original material format. Drilling specimens from larger HA disks was substantially faster - by a factor of two - than from biological material of the same size, as no reorientation of the drill is necessary between position changes. However, performing embedding and surface grinding still takes the same amount of time as for biological material. While this was required for consistency in this experiment, it could be possible for future experiments to use pre-formed HA disks of suitable size, for zero preparation time. It may be an interesting proposition for manufacturers to vary the composition of HA disks to include additional chemical substitutions in an attempt to make them even more representative of organic enamel samples.

\section{Physical similarity and other factors}

Physical similarity refers to the characteristics of the specimen post-erosion, which can be measured by physical methods, e.g. X-Ray, 3D scanning or needle-based profilometry. Roughness of the disk, depth of erosion as well as the actual erosion pattern would fall under this category, and were assessed by profilometry in this case.

In past experiments, human enamel exposed to a laminar $\mathrm{HCl}$-flow has displayed a relatively smooth eroded surface with deviations on the order of zero to five $\mu \mathrm{m}$, and a slanted profile inclined in parallel from the direction of flow. Profilometry of the HA specimens implied that HA disks indeed experience a similar sloping profile. A volume calculation based on the average depth of the erosion cavity, area of the sample, and calcium content of pure HA verified that the calcium measurement was in the similar order of magnitude. In terms of differences, however, the HA profile showed higher roughness levels over the enamel specimens. This can be seen in the isolated mini-peaks ( $\mu \mathrm{m}$-size deviations) in the otherwise smooth surface as presented in Figure 2, and may indicate larger crystallites breaking away from the pressed HA structure during flow than from human enamel.

Physically, the surface of HA displays substantial differences both in terms of hardness as well as elasticity. Based on the experience from grinding the specimens, HA surfaces in all likelihood do not resist wear and tear like those from enamel, indicating that it is probably not a suitable candidate for the substitution or benchmarking in experiments involving 
mechanical strain, i.e. experiments relying primarily on physically measured characteristics such as hardness or elastic modulus.

\section{Further research}

While this experiment allows characterising the surface chemistry of HA under flow conditions, it would also be interesting to extend HA testing to a combination of longer-term acid exposure and artificial remineralisation, i.e. 'artificial caries' induction such as presented by Schmidlin et al. [33]. This type of experiment relies on transverse microradiography for tracking the spread of artificial carious lesions, where the uniform density of HA could allow for less noisy X-ray imaging - if indeed artificial caries could be induced.

Further research should also involve a low-variance benchmarking of different acidic solutions and a wider range of $\mathrm{pH}$ values with the same experimental procedure, in order to establish confidence intervals for correction constants between pure HA and human enamel, which may eventually allow full substitution. In addition, pre-formed as opposed to drilled HA specimens could be employed to decrease test preparation time, and subjected to a wider range of chemical erosion experiments, such as static acid exposure and remineralisation. Future studies should also include human saliva in the experimental set-up, which constitutes an essential clinical factor in the development of erosions.

\section{Conclusion}

The null-hypothesis that HA would display erosion behaviour similar to that of human and bovine enamel specimens, at lower experimental variability, was rejected. The range of calcium dissolution rates of HA overlapped with human enamel at the lower two $\mathrm{pH}$ levels, however difference between means could be detected statistically at $\mathrm{pH} 2.0$ and 3.0, with a general tendency to erode less than biologic enamel. Measurement variability was consistently lower. This combination of traits implies that HA may not be suitable for full substitution in studies focusing on absolute values of measurements, but is still useful for establishing consistent relative trends. 


\section{References}

[1] T. Imfeld, Dental erosion. Definition, classification and links, Eur J Oral Sci. 104 (1996) 151-155.

[2] A. Lussi, C. Ganss (Eds.), Monographs in Oral Science, Vol. 25, Erosive Tooth Wear. From Diagnosis to Therapy, second, revised and extended edition, Karger, Basel, 2014.

[3] T. Jaeggi, A. Lussi, Prevalence, incidence and distribution of erosion, Monogr Oral Sci. 25 (2014) 55-73. https://doi.org/10.1159/000360973.

[4] A. Lussi, T. Jaeggi, D. Zero, The role of diet in the aetiology of dental erosion, Caries Res. 38 Suppl 1 (2004) 34-44. https://doi.org/10.1159/000074360.

[5] P. Scheutzel, Etiology of dental erosion--intrinsic factors, Eur J Oral Sci. 104 (1996) 178-190.

[6] D.L. Gambon, H.S. Brand, E.C. Veerman, Dental erosion in the 21st century: what is happening to nutritional habits and lifestyle in our society, Br Dent J. 213 (2012) 55-57. https://doi.org/10.1038/sj.bdj.2012.613.

[7] L.H. Eusebi, R. Ratnakumaran, Y. Yuan, M. Solaymani-Dodaran, F. Bazzoli, A.C. Ford, Global prevalence of, and risk factors for, gastro-oesophageal reflux symptoms: a meta-analysis, Gut. 67 (2018) 430-440. https://doi.org/10.1136/gutjnl-2016-313589.

[8] A. Keski-Rahkonen, L. Mustelin, Epidemiology of eating disorders in Europe: prevalence, incidence, comorbidity, course, consequences, and risk factors, Curr Opin Psychiatry. 29 (2016) 340-345. https://doi.org/10.1097/YCO.0000000000000278.

[9] M. Nagl, C. Jacobi, M. Paul, K. Beesdo-Baum, M. Höfler, R. Lieb, H.U. Wittchen, Prevalence, incidence, and natural course of anorexia and bulimia nervosa among adolescents and young adults, Eur Child Adolesc Psychiatry. 25 (2016) 903-918. https://doi.org/10.1007/s00787-015-0808-z.

[10] R.P. Shellis, C. Ganss, Y. Ren, D.T. Zero, A. Lussi, Methodology and models in erosion research: discussion and conclusions, Caries Res. 45 Suppl 1 (2011) 69-77. https://doi.org/10.1159/000325971.

[11] J. Rees, T. Loyn, B. Chadwick, Pronamel and tooth mousse: an initial assessment of erosion prevention in vitro, J Dent. 35 (2007) 355-357. 10.1016/j.jdent.2006.10.005.

[12] M.C. Huysmans, A. Young, C. Ganss, The role of fluoride in erosion therapy, Monogr Oral Sci. 25 (2014) 230-243. https://doi.org/10.1159/000360555.

[13] A. Aykut-Yetkiner, T. Attin, A. Wiegand, Prevention of dentine erosion by brushing with anti-erosive toothpastes, J Dent. 42 (2014) 856-861. https://doi.org/10.1016/j.jdent.2014.03.011.

[14] T. Attin, F. Wegehaupt, D. Gries, A. Wiegand, The potential of deciduous and permanent bovine enamel as substitute for deciduous and permanent human enamel: Erosion-abrasion experiments, J Dent. 35 (2007) 773-777. https://doi.org/10.1016/j.jdent.2007.07.007.

[15] M. Sydney-Zax, I. Mayer, D. Deutsch, Carbonate content in developing human and bovine enamel, J Dent Res. 70 (1991) 913-916. https://doi.org/10.1177/00220345910700051001.

[16] J.C. Elliot, Structure, crystal chemistry and density of enamel apatites, Ciba Foundation Symposium 205 - dental enamel. (1997) 54-72.

[17] T. Attin, K. Becker, C. Hannig, W. Buchalla, R. Hilgers, Method to detect minimal amounts of calcium dissolved in acidic solutions, Caries Res. 39 (2005) 432-436. https://doi.org/10.1159/000086852.

[18] D.A. Lazarchik, S.J. Filler, Effects of gastroesophageal reflux on the oral cavity, Am J Med. 103 (1997) 107S-113S. 
[19] D.W. Bartlett, P.Y. Coward, Comparison of the erosive potential of gastric juice and a carbonated drink in vitro, J Oral Rehabil. 28 (2001) 1045-1047.

[20] T. Attin, K. Becker, A. Wiegand, T.T. Tauböck, F.J. Wegehaupt, Impact of laminar flow velocity of different acids on enamel calcium loss, Clin Oral Investig. 17 (2013) 595-600. https://doi.org/10.1007/s00784-012-0731-3.

[21] A. Wiegand, W. Meier, E. Sutter, A.C. Magalhães, K. Becker, M. Roos, T. Attin, Protective effect of different tetrafluorides on erosion of pellicle-free and pelliclecovered enamel and dentine, Caries Res. 42 (2008) 247-254. https://doi.org/10.1159/000135669.

[22] C. Hannig, A. Hamkens, K. Becker, R. Attin, T. Attin, Erosive effects of different acids on bovine enamel: release of calcium and phosphate in vitro, Arch Oral Biol. 50 (2005) 541-552. https://doi.org/10.1016/j.archoralbio.2004.11.002.

[23] R Core Team: A language and environment for statistical computing. R Foundation for Statistical Computing. https://www.R-project.org/, (2015)

[24] T. Pohlert, The Pairwise Multiple Comparison of Mean Ranks Package (PMCMR). R package, (2014)

[25] R.P. Shellis, M.E. Barbour, S.B. Jones, M. Addy, Effects of $\mathrm{pH}$ and acid concentration on erosive dissolution of enamel, dentine, and compressed hydroxyapatite., Eur J Oral Sci. 118 (2010) 475-482. https://doi.org/10.1111/j.1600-0722.2010.00763.x .

[26] C. Xu, R. Reed, J.P. Gorski, Y. Wang, M.P. Walker, The Distribution of Carbonate in Enamel and its Correlation with Structure and Mechanical Properties, J Mater Sci. 47 (2012) 8035-8043. https://doi.org/10.1007/s10853-012-6693-7.

[27] J.M. ten Cate, J.D. Featherstone, Mechanistic aspects of the interactions between fluoride and dental enamel, Crit Rev Oral Biol Med. 2 (1991) 283-296.

[28] J.D. Featherstone, The science and practice of caries prevention, J Am Dent Assoc. 131 (2000) 887-899.

[29] P.R. Patel, W.E. Brown, Thermodynamic solubility product of human tooth enamel: powdered sample, J Dent Res. 54 (1975) 728-736. https://doi.org/10.1177/00220345750540040601.

[30] G.H. Yassen, J.A. Platt, A.T. Hara, Bovine teeth as substitute for human teeth in dental research: a review of literature, J Oral Sci. 53 (2011) 273-282.

[31] F. Wegehaupt, D. Gries, A. Wiegand, T. Attin, Is bovine dentine an appropriate substitute for human dentine in erosion/abrasion tests, J Oral Rehabil. 35 (2008) 390394. https://doi.org/10.1111/j.1365-2842.2007.01843.x.

[32] A.J. White, C. Yorath, V. ten Hengel, S.D. Leary, M.C. Huysmans, M.E. Barbour, Human and bovine enamel erosion under 'single-drink' conditions, Eur J Oral Sci. 118 (2010) 604-609. https://doi.org/10.1111/j.1600-0722.2010.00779.x.

[33] P.R. Schmidlin, B. Sener, T. Attin, A. Wiegand, Protection of sound enamel and artificial enamel lesions against demineralisation: caries infiltrant versus adhesive, $\mathrm{J}$ Dent. 40 (2012) 851-856. https://doi.org/10.1016/j.jdent.2012.07.003. 


\section{Tables}

\section{Table 1}

Mean calcium release (Mean $\mathrm{Ca}[\mathrm{nmol} / 10 \mathrm{~s}]$ ) of the three tested specimens with their mean coefficient of variation (\%). Further, results of the pairwise statistical comparison (using Conover's test posthoc to the Kruskal-Wallis test) to the human specimens are given.

\begin{tabular}{|c|c|c|c|c|}
\hline & & Human & Bovine & HA \\
\hline N (spec & ens) & 18 & 18 & 18 \\
\hline N (mea & ements per pH) & 180 & 180 & 180 \\
\hline pH 2.0 & Mean Ca [nmol/10 s] & 10.04 & 10.39 & 8.85 \\
\hline & $\begin{array}{l}\text { Stat. sig. difference } \\
\text { (compared to human series) }\end{array}$ & - & $p=0.79$ & $p<0.05$ \\
\hline pH 2.6 & Mean Ca [nmol/10 s] & 2.42 & 2.54 & 2.48 \\
\hline & $\begin{array}{l}\text { Stat. sig. difference } \\
\text { (compared to human series) }\end{array}$ & - & $p=0.34$ & $p=0.70$ \\
\hline pH 3.0 & Mean Ca [nmol/10 s] & 1.27 & 1.24 & 1.06 \\
\hline & $\begin{array}{l}\text { Stat. sig. difference } \\
\text { (compared to human series) }\end{array}$ & - & $p=0.80$ & $p<0.05$ \\
\hline Mean c & ficient of variation & $25 \%$ & $25 \%$ & $17 \%$ \\
\hline
\end{tabular}

Table 2

Percentiles of the calcium release $[\mathrm{nmol} / 10 \mathrm{~s}]$ of the human and bovine enamel specimens as well as the hydroxyapatite specimens at the three tested $\mathrm{pH}$ levels.

\begin{tabular}{|c|c|c|c|c|c|c|}
\hline \multicolumn{7}{|c|}{ Ca release in $\mathrm{nmol} / 10 \mathrm{~s}$} \\
\hline \multicolumn{2}{|c|}{ [Percentile] } & \multirow{2}{*}{$\begin{array}{r}\mathbf{0 \%} \\
3.70\end{array}$} & \multirow{2}{*}{$\begin{array}{l}\mathbf{2 5 \%} \\
8.87\end{array}$} & \multirow{2}{*}{\begin{tabular}{|l}
$\mathbf{5 0 \%}$ \\
10.56
\end{tabular}} & \multirow{2}{*}{$\begin{array}{l}\mathbf{7 5 \%} \\
10.96 \\
\end{array}$} & \multirow{2}{*}{$\begin{array}{c}\mathbf{1 0 0 \%} \\
14.06\end{array}$} \\
\hline pH 2.0 & Human & & & & & \\
\hline & Bovine & 5.84 & 9.87 & 10.40 & 10.83 & 14.92 \\
\hline & HA & 5.90 & 7.75 & 8.70 & 10.03 & 11.70 \\
\hline \multirow[t]{3}{*}{ pH 2.6} & Human & 1.01 & 1.80 & 2.38 & 2.80 & 3.84 \\
\hline & Bovine & 0.76 & 2.10 & 2.59 & 2.80 & 4.28 \\
\hline & HA & 2.00 & 2.25 & 2.50 & 2.58 & 3.80 \\
\hline \multirow[t]{3}{*}{ pH 3.0 } & Human & 0.73 & 1.19 & 1.29 & 1.41 & 1.61 \\
\hline & Bovine & 0.60 & 1.22 & 1.30 & 1.39 & 1.69 \\
\hline & HA & 0.70 & 0.99 & 1.07 & 1.14 & 1.55 \\
\hline
\end{tabular}




\section{Figures}

\section{Figure legend 1}

Calcium release [nmol/10 s] of the human and bovine enamel specimens as well as the hydroxyapatite specimens at the three tested $\mathrm{pH}$ levels.

\section{Figure legend 2}

Above: Single-track depth profile of a HA sample pre- (black) and post-erosion (white).

Below: Resulting difference curve of pre- and post-erosion profile. 